\author{
Craig C. Mello ${ }^{1,2}$ \& Darryl Conte $\mathrm{Jr}^{2}$
}

${ }^{1}$ Howard Hughes Medical Institute and ${ }^{2}$ Program in Molecular Medicine, University of Massachusetts Medical School, Worcester, Massachusetts 01605, USA (e-mail: craig.mello@umassmed.edu)

The recent discoveries of RNA interference and related RNA silencing pathways have revolutionized our understanding of gene regulation. RNA interference has been used as a research tool to control the expression of specific genes in numerous experimental organisms and has potential as a therapeutic strategy to reduce the expression of problem genes. At the heart of RNA interference lies a remarkable RNA processing mechanism that is now known to underlie many distinct biological phenomena.

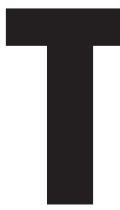

he term 'RNA world' was first coined to describe a hypothetical stage in the evolution of life some four billion years ago when RNA may have been the genetic material and catalyst for emerging life on Earth ${ }^{1,2}$. This original RNA world, if it ever existed on Earth, is long gone. But this Insight deals with a process that reflects an RNA world that is alive and thriving within our cells - RNA silencing or RNA interference (RNAi). When exposed to foreign genetic material (RNA or DNA), many organisms mount highly specific counter attacks to silence the invading nucleic-acid sequences before these sequences can integrate into the host genome or subvert cellular processes. At the heart of these sequencedirected immunity mechanisms is double-stranded RNA (dsRNA). Interestingly, dsRNA does more than help to defend cells against foreign nucleic acids - it also guides endogenous developmental gene regulation, and can even control the modification of cellular DNA and associated chromatin. In some organisms, RNAi signals are transmitted horizontally between cells and, in certain cases, vertically through the germ line from one generation to the next. The reviews in this Insight show our progress in understanding the mechanisms that underlie RNA-mediated gene regulation in plants and animals, and detail current efforts to harness this mechanism as a research tool and potential therapy. Here we introduce the world of RNAi, and provide a brief overview of this rapidly growing field.

\section{Discovering the trigger}

Crucial to understanding a gene-silencing mechanism such as RNAi is knowing how to trigger it. This is important from the theoretical perspective of understanding a remarkable biological response (see review in this issue by Meister and Tuschl, page 343); but it also has obvious practical ramifications for using the silencing mechanism as an experimental tool (see review in this issue by Hannon and Rossi, page 371). The observation by Fire et al. ${ }^{3}$ that dsRNA is a potent trigger for RNAi in the nematode Caenorhabditis elegans (Fig. 1) was important because it immediately suggested a simple approach for efficient induction of gene silencing in C. elegans and other organisms, and accelerated the discovery of a unifying mechanism that underlies a host of cellular and developmental pathways. However, there were substantial barriers to the acceptance of the idea that dsRNA could trigger sequence-specific gene silencing.

First, at the time, dsRNA was thought to be a nonspecific silencing agent that triggers a general destruction of messenger RNAs and the complete suppression of protein translation in mammalian cells ${ }^{4,5}$. Second, dsRNA is energetically stable and inherently incapable of further specific Watson-Crick base pairing. So a model in which dsRNA activates sequence-specific silencing implies the existence of cellular mechanisms for unwinding the dsRNA and promoting the search for complementary base-pairing partners among the vast pool of cellular nucleic-acid sequences. Hypotheses that require a paradigm shift and depend on the existence of a whole set of hitherto unknown activities are rarely appealing.

So why was dsRNA proposed as a trigger for RNAi and why was this idea so rapidly accepted? To answer this question we must make a brief historical digression. In 1995, Guo and Kemphues $^{6}$ attempted to use RNA complementary to the C. elegans par-1 mRNA to block par-1 expression. This technique is known as 'antisense-mediated silencing', whereby large amounts of a nucleic acid whose sequence is complementary to the target messenger RNA are delivered into the cytoplasm of a cell. Base pairing between the 'sense' mRNA sequence and the complementary 'antisense' interfering nucleic acid is thought to passively block the processing or translation of mRNA, or result in the recruitment of nucleases that promote mRNA destruction ${ }^{7,8}$. To their surprise, Guo and Kemphues found that both the antisense and the control sense RNA preparations induced silencing. Sense RNA is identical to the mRNA and so cannot base pair with the mRNA to cause interference, raising the question of how this RNA could induce silencing. Was an active silencing response being triggered against the foreign RNA, regardless of its polarity? Or was the silencing apparently induced by sense RNA actually mediated by antisense RNA? (Antisense RNA was known to contaminate the type of in vitro transcription products used in these assays.) Despite confusion about the nature of the RNA that triggered the phenomenon, this so-called antisense-mediated silencing method continued to be used to silence genes in C. elegans.

More surprises were in store. While using this antisense technique to silence C. elegans genes, we were amazed to find that the silencing effect could be transmitted in the germ line $e^{3}$. A remarkably potent silencing signal could be passed through the sperm or the egg for up to several generations $s^{3,9}$. Equally remarkable, the silencing effect could also spread from tissue to tissue within the injected animal ${ }^{3}$. Taken together, the apparent lack of strand specificity, the remarkable potency of the RNA trigger, and the systemic spread and inheritance properties of the silencing phenomenon prompted the creation of a new term, $\mathrm{RNAi}^{10}$. Importantly, the properties of RNAi demanded the existence of cellular 


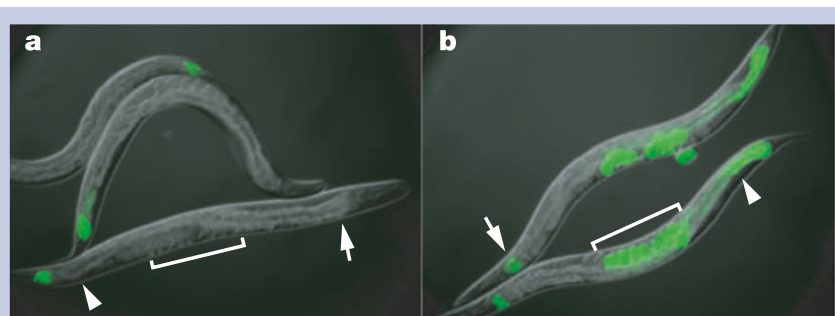

Figure 1 RNAi in C. elegans. Silencing of a green fluorescent protein (GFP) reporter in C. elegans occurs when animals feed on bacteria expressing GFP dsRNA (a) but not in animals that are defective for RNAi (b). Note that silencing occurs throughout the body of the animal, with the exception of a few cells in the tail that express some residual GFP. The signal is lost in intestinal cells near the tail (arrowhead) as well as near the head (arrow). The lack of GFP-positive embryos in a (bracketed region) demonstrates the systemic spread and inheritance of silencing.

mechanisms that initiate and amplify the silencing signal, and led us to suggest that the RNAi mechanism represents an active organismal response to foreign $\mathrm{RNA}^{3}$.

Although our initial models saw dsRNA as an intermediate in the amplification of the silencing signal, Fire ${ }^{3}$ suggested that dsRNA, which is often encountered by cells during viral infection, might itself be the initial trigger. In this model, instead of antisense RNA passively initiating silencing by pairing with the target mRNA, the presence of low concentrations of both sense and antisense strands in the RNA preparation was proposed to result in small amounts of dsRNA: on introduction into the animal, this dsRNA could be recognized as foreign, thereby activating cellular amplification and inheritance mechanisms. Because it was possible to produce and purify in vitro synthesized RNA and introduce it directly into C. elegans without the need for transgene-driven expression, this theory was easily tested. dsRNA proved to be an extremely potent activator of RNAi - at least 10 -fold and perhaps 100 -fold more effective than purified preparations of single-stranded $\mathrm{RNA}^{3}$.

\section{Taking the biological world by storm}

With the discovery of an extremely potent trigger for RNAi, it became possible to expose large populations of animals to dsRNA: animals were soaked in dsRNA ${ }^{11}$ or given food containing bacterially expressed dsRNA ${ }^{12,13}$. By facilitating genetic screens, these methods led to the identification of many C. elegans genes required for $\mathrm{RNAi}^{14}$. Comparison of the $C$. elegans genes required for RNAi to genes required for gene silencing in Drosophila ${ }^{15,16}$, plants ${ }^{17}$ and fungi ${ }^{18}$ confirmed that the silencing phenomena known variously as posttranscriptional gene silencing (PTGS $)^{19}$, co-suppression ${ }^{20}$, quelling ${ }^{21}$ and RNAi, share a common underlying mechanism that reflects an ancient origin in a common ancestor of fungi, plants and animals. This realization was followed by a flurry of exciting results: dsRNA was shown to induce silencing in Drosophila ${ }^{22}$, and in a host of other organisms including organisms that were otherwise unsuited to genetic analysis ${ }^{23,24}$. Small RNAs were shown to be produced in plants undergoing PTGS $^{25}$, and were identified as the common currency of RNA silencing pathways ${ }^{26-28}$ (see review in this issue by Baulcombe, page 356). The dsRNA-processing enzyme Dicer $^{29}$ was found to produce these small RNAs, now called short interfering RNAs (siRNAs). Synthetic RNAs engineered to look like the products of Dicer were shown to induce sequence-specific gene silencing in human cells without initiating the nonspecific gene silencing pathways ${ }^{30}$. A class of natural hairpin dsRNAs ${ }^{31,32}$, now called microRNAs (miRNAs; see review in this issue by Ambros, page 350), was shown to be processed by Dicer ${ }^{33-35}$ and to function together with RDE-1 homologues ${ }^{35}$, thereby linking the RNAi machinery to a natural developmental gene regulatory mechanism. Finally, more recently, the RNAi machinery was linked to chromatin regulation in yeast ${ }^{36}$, and to chromosomal rearrangement during development of the somatic macronucleus in
Tetrahymena ${ }^{37}$. These and other breakthroughs united previously disparate fields by identifying a common core mechanism that involves the processing of dsRNA into small RNA-silencing guides (Fig. 2). In short, dsRNA had taken the biological world by storm.

\section{Other silencing triggers}

Although it was clear that dsRNA was important either as a silencing trigger or as an intermediate in all the RNAi-related silencing pathways, it was not known whether other stimuli (besides dsRNA) could trigger silencing. For example, silencing in response to a DNA transgene could still involve a dsRNA trigger: the transgene might integrate itself into the genome in such a way that a nearby promoter, or an inverted copy of the transgene itself, leads to the production of dsRNA, which could in turn enter directly into the RNAi pathway. Consistent with this idea, transgenes engineered to express both sense and antisense strands of a gene in plants can lead to efficient silencing, which is more reproducible and robust than that achieved by transgenes expressing either strand alone ${ }^{38}$.

But several lines of evidence suggest that transgenes can trigger silencing through mechanisms not involving a dsRNA trigger (Fig. 2). A key gene family involved in silencing pathways in plants ${ }^{39,40}$, fungi ${ }^{41}$ and C. elegans $s^{42,43}$ contains genes that encode putative cellular RNAdependent RNA polymerases (RdRPs; also known as RDRs). Members of this family of proteins were identified in forward genetic screens - whereby mutant genes are isolated from an organism showing abnormal phenotypic characteristics - as factors required for co-suppression in plants and quelling in Neurospora. (Co-suppression results from post-transcriptional silencing of both a transgene and the endogenous copies of the corresponding cellular gene.) Interestingly, although cellular RdRP genes were required for transgene-mediated co-suppression in plants ${ }^{39,40}$, they were not essential for virus-induced silencing of a transgene $e^{39}$, presumably because the virus provides its own viral RNA polymerase. Furthermore, RdRPs have been shown to direct primer-independent synthesis of complementary RNA ${ }^{44,45}$. Together, these findings suggest that the transgene or its single-stranded mRNA products could be the original stimulus for co-suppression and quelling. In this type of silencing, the RdRP somehow recognizes transgene products as abnormal or 'aberrant' and subsequently converts this initial silencing trigger into dsRNA ${ }^{46,47}$. In this case, the dsRNA is an intermediate in the silencing pathway rather than the trigger. The RdRP-derived dsRNA is then likely to be processed by Dicer and to enter downstream silencing complexes that are similar, or identical, to those formed in response to a dsRNA trigger.

But how might the transgene mRNA be recognized as foreign? The answer to this question is not known. Hence, this 'aberrant transcript' model has, perhaps undeservedly, received little attention of late. One possibility discussed by Baulcombe (review in this issue, page 356) is that high levels of expression of the transgene mRNA leads to the accumulation of mRNA-processing defects (for example, non-polyadenylated transcripts) that are somehow recognized by the RdRP. Alternatively, the transgene DNA or the chromatin itself may be 'marked' for silencing by the cell. When initially delivered to cells, the transgene DNA could be recognized as foreign owing to its lack of associated proteins. During the rapid assembly of naked DNA into chromatin $^{48}$, the host cell may, in self-defence, somehow mark the transgene chromatin so that RdRP is recruited. RdRP acting on nascent transcripts could then result in dsRNA formation and subsequent silencing. Consistent with this possibility, fission yeast RdRP was found to physically associate with silent heterochromatin ${ }^{36}$. Despite the mysterious nature of the silencing mark recognized by RdRPs, it seems likely that, at least in some cases, RdRPs may produce dsRNA that functions as an intermediate rather than as the primary trigger for silencing.

Genetic studies suggest that distinct silencing triggers may also exist in C. elegans. Both RDE-1 and the dsRNA-binding protein RDE-4 (ref. 49) are essential for mediating the silencing induced by 


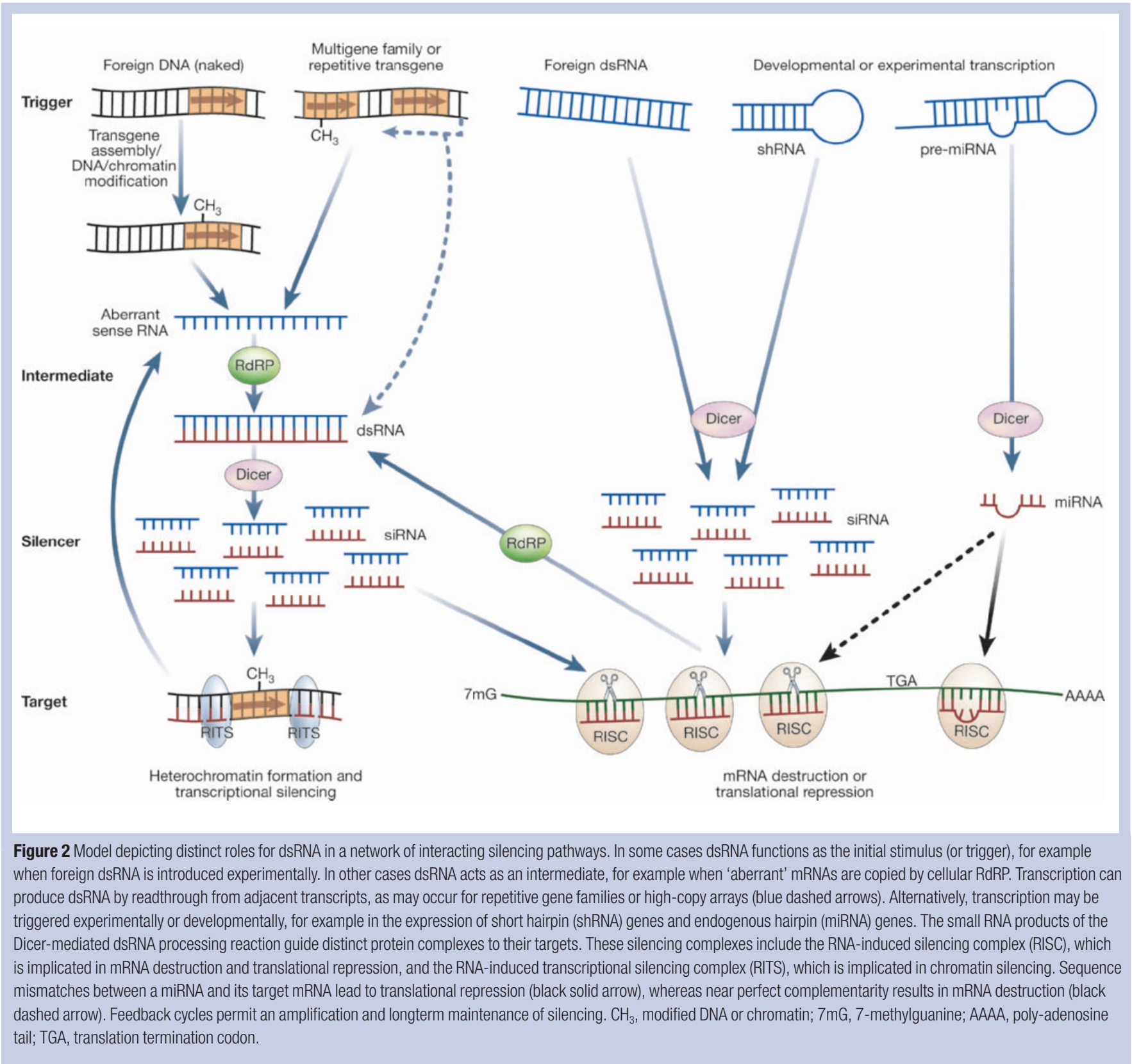

injecting, feeding or expressing dsRNA ${ }^{14}$. However, RDE-1 and RDE-4 are not required for transposon silencing or for co-suppression ${ }^{14,50,51}$. Furthermore, RDE- 1 and RDE- 4 are not required for the inheritance of RNAi-induced silencing' ${ }^{9}$, which suggests that they are only required during the initial exposure to dsRNA. These findings indicate that transposon silencing and co-suppression in C. elegans are initiated by means of distinct triggers. As discussed above, an appealing idea is that a chromatin 'signature' stimulates the production of aberrant transcripts and the formation of a novel species of dsRNA (perhaps nuclear) that is distinct from the dsRNA that initiates silencing by means of RDE- 1 and RDE-4. Again, in this model the initial trigger is the chromatin structure of the transposon locus or the transgene, and dsRNA acts as an intermediate in the silencing pathway (Fig. 2). Perhaps a similar RdRP-derived dsRNA functions in the RDE-1- and RDE-4-independent mechanisms that propagate silencing from one generation to the next.

Ten years ago, de novo cytosine methylation of genomic DNA was shown to occur in plants infected with RNA viroids whose sequences were homologous to the methylated genomic sequences ${ }^{52}$. This process was referred to as RNA-directed DNA methylation (RdDM).
Subsequently, dsRNA targeting a promoter was shown to trigger RdDM and initiate transcriptional silencing. The silencing was accompanied by the production of siRNAs $s^{53}$, pointing to an RNAilike mechanism for the initiation of transcriptional gene silencing. Recent work in fission yeast has now convincingly demonstrated that the formation of silent heterochromatin can be guided by small $\mathrm{RNAs}^{54}$ and the RNA-silencing machiner $\mathrm{y}^{36}$. In Drosophila, the RNAsilencing machinery was also required for heterochromatin formation and for silencing multicopy transgenes and pericentric DNA ${ }^{55}$. The discovery of an underlying molecular connection between RNA guides and chromatin remodelling has been one of the most exciting recent developments in the field of epigenetics. It is becoming clear that RNAi has an important role in the initiation of heterochromatin formation and transcriptional silencing in plants, fungi and animals (see review in this issue by Lippman and Martienssen, page 364).

The possibility of feedback between RNAi, its potential chromatinassociated trigger, and chromatin-mediated silencing maintenance mechanisms raises further questions about the ultimate causes of silencing. For example, were C. eleganstransposons originally silenced by means of an RDE-1/RDE-4-dependent dsRNA signal, resulting 
from sense and antisense readthrough transcription from insertion points in the genome ${ }^{56,57}$ ? Perhaps over time this initial dsRNAtriggered silencing signal was replaced and augmented by a chromatin-associated silencing-maintenance signal.

\section{Outlook for the RNA world}

The numerous branching and converging silencing pathways that seem to exist in diverse organisms will no doubt require many years of research to unravel. It is already clear that different organisms have evolved distinct mechanisms, or at least variations on a common theme. In some cases, differences seem to exist in the extent to which silencing relies on a particular mode of regulation. For example, plants show a preponderance of miRNA-guided mRNA cleavage ${ }^{58,59}$, but only one example of this mode of regulation has been found in animals ${ }^{60}$. The diversity of RNA silencing phenomena suggests that other interesting findings await discovery. For example, the existence of an inheritance mechanism for the transmission of RNAi in C.elegans raises the question of whether natural small RNAs are transmitted in germ cells or other developmental cell lineages in other animals, including humans. Extrachromosomal inheritance of silencing patterns by means of small RNAs could provide sophisticated layers of gene regulation, at both post-transcriptional and chromatinmodifying levels. These small RNAs may be important in stem-cell maintenance and development, and differential localization of such RNAs may have a role in the generation of cellular diversity. It will be interesting to discover if the phenomenon of lateral transport of RNA from cell to cell, so far observed in plants ${ }^{61,62}$ and C. elegans, is more widespread. As well as having a role in immunity, could 'epigenetic RNA morphogens' allow cells to modulate the activity of developmentally important genes or mRNAs in neighbouring cells? This type of regulation might be particularly useful when cells, such as neurons, communicate at junctions that are far from the cell nucleus.

The past ten years have seen an explosion in the number of noncoding RNAs found to orchestrate remarkably diverse functions ${ }^{63,64}$. These functions include: sequence-specific modification of cellular RNAs guided by small nucleolar RNAs ${ }^{65}$; induction of chromosomewide domains of chromatin condensation by the mammalian noncoding RNA Xist (X-inactive specific transcript $)^{66}$; autosomal gene imprinting and silencing by noncoding mammalian Air (antisense IgF2rRNA) ${ }^{67}$; and finally sequence-directed cleavage and/or repression of target mRNAs and genes by miRNAs and siRNAs, discussed here and in the accompanying reviews. Some have likened this period to an RNA revolution. But considering the potential role of RNA as a primordial biopolymer of life, it is perhaps more apt to call it an RNA 'revelation'. RNA is not taking over the cell - it has been in control all along. We just didn't realize it until now.

doi:10.1038/nature02872

1. Joyce, G. F. \& Orgel, L. E. in The RNA World (eds Gestland, R. F., Cech, T. R. \& Atkins, J. F.) 49-77 (Cold Spring Harbor Laboratory Press, New York, 1999).

2. Gilbert, W. The RNA world. Nature 319, 618 (1986).

3. Fire, A. et al. Potent and specific genetic interference by double-stranded RNA in Caenorhabditis elegans. Nature 391, 806-811 (1998).

4. Proud, C. G. PKR: a new name and new roles. Trends Biochem. Sci. 20, 241-246 (1995).

5. Williams, B. R. Role of the double-stranded RNA-activated protein kinase (PKR) in cell regulation. Biochem. Soc. Trans. 25, 509-513 (1997).

6. Guo, S. \& Kemphues, K. J. par-1, a gene required for establishing polarity in C. elegans embryos, encodes a putative Ser/Thr kinase that is asymmetrically distributed. Cell 81, 611-620 (1995).

7. Nellen, W. \& Lichtenstein, C. What makes an mRNA anti-sense-itive? Trends Biochem. Sci. 18, 419-423 (1993).

8. Izant, J. G. \& Weintraub, H. Inhibition of thymidine kinase gene expression by anti-sense RNA: a molecular approach to genetic analysis. Cell 36, 1007-1015 (1984).

9. Grishok, A., Tabara, H. \& Mello, C. C. Genetic requirements for inheritance of RNAi in C. elegans. Science 287, 2494-2497 (2000).

10. Rocheleau, C. E. et al. Wnt signaling and an APC-related gene specify endoderm in early C. elegans embryos. Cell 90, 707-716 (1997).

11. Tabara, H., Grishok, A. \& Mello, C. C. RNAi in C. elegans: soaking in the genome sequence. Science 282, 430-431 (1998).

12. Timmons, L. \& Fire, A. Specific interference by ingested dsRNA. Nature 395, 854 (1998).

13. Timmons, L., Court, D. L. \& Fire, A. Ingestion of bacterially expressed dsRNAs can produce specific and potent genetic interference in Caenorhabditis elegans. Gene 263, 103-112 (2001).
14. Tabara, H. et al. The rde-1 gene, RNA interference, and transposon silencing in C. elegans. Cell 99, 123-132 (1999).

15. Schmidt, A. et al. Genetic and molecular characterization of sting, a gene involved in crystal formation and meiotic drive in the male germ line of Drosophila melanogaster. Genetics 151, 749-760 (1999).

16. Aravin, A. A. et al. Double-stranded RNA-mediated silencing of genomic tandem repeats and transposable elements in the D. melanogaster germline. Curr. Biol. 11, 1017-1027 (2001).

17. Fagard, M., Boutet, S., Morel, J. B., Bellini, C. \& Vaucheret, H. AGO1, QDE-2, and RDE-1 are related proteins required for post-transcriptional gene silencing in plants, quelling in fungi, and RNA interference in animals. Proc. Natl Acad. Sci. USA 97, 11650-11654 (2000).

18. Catalanotto, C., Azzalin, G., Macino, G. \& Cogoni, C. Gene silencing in worms and fungi. Nature 404, 245 (2000).

19. de Carvalho, F. et al. Suppression of beta-1,3-glucanase transgene expression in homozygous plants. EMBO J. 11, 2595-2602 (1992).

20. Napoli, C., Lemieux, C. \& Jorgensen, R. Introduction of a chimeric chalcone synthase gene into petunia results in reversible co-suppression of homologous genes in trans. Plant Cell 2, 279-289 (1990).

21. Romano, N. \& Macino, G. Quelling: transient inactivation of gene expression in Neurospora crassa by transformation with homologous sequences. Mol. Microbiol. 6, 3343-3353 (1992).

22. Kennerdell, J. R. \& Carthew, R. W. Use of dsRNA-mediated genetic interference to demonstrate that frizzled and frizzled 2 act in the wingless pathway. Cell 95, 1017-1026 (1998).

23. Ngo, H., Tschudi, C., Gull, K. \& Ullu, E. Double-stranded RNA induces mRNA degradation in Trypanosoma brucei. Proc. Natl Acad. Sci. USA 95, 14687-14692 (1998).

24. Bosher, J. M. \& Labouesse, M. RNA interference: genetic wand and genetic watchdog. Nature Cell Biol. 2, E31-E36 (2000).

25. Hamilton, A. J. \& Baulcombe, D. C. A species of small antisense RNA in post-transcriptional gene silencing in plants. Science 286, 950-952 (1999).

26. Hammond, S. M., Bernstein, E., Beach, D. \& Hannon, G. J. An RNA-directed nuclease mediates posttranscriptional gene silencing in Drosophila cells. Nature 404, 293-296 (2000).

27.Zamore, P. D., Tuschl, T., Sharp, P. A. \& Bartel, D. P. RNAi: double-stranded RNA directs the ATPdependent cleavage of mRNA at 21 to 23 nucleotide intervals. Cell 101, 25-33 (2000).

28. Parrish, S. \& Fire, A. Distinct roles for RDE-1 and RDE-4 during RNA interference in Caenorhabditis elegans. RNA 7, 1397-1402 (2001).

29. Bernstein, E., Caudy, A. A., Hammond, S. M. \& Hannon, G. J. Role for a bidentate ribonuclease in the initiation step of RNA interference. Nature 409, 363-366 (2001).

30. Elbashir, S. M. et al. Duplexes of 21-nucleotide RNAs mediate RNA interference in cultured mammalian cells. Nature 411, 494-498 (2001).

31. Reinhart, B. J. et al. The 21-nucleotide let-7 RNA regulates developmental timing in Caenorhabditis elegans. Nature 403, 901-906 (2000).

32. Lee, R. C., Feinbaum, R. L. \& Ambros, V. The C. elegans heterochronic gene lin-4 encodes small RNAs with antisense complementarity to lin-14. Cell 75, 843-854 (1993).

33. Hutvágner, G. et al. A cellular function for the RNA-interference enzyme Dicer in the maturation of the let-7 small temporal RNA. Science 293, 834-838 (2001).

34. Ketting, R. F. et al. Dicer functions in RNA interference and in synthesis of small RNA involved in developmental timing in C. elegans. Genes Dev. 15, 2654-2659 (2001).

35. Grishok, A. et al. Genes and mechanisms related to RNA interference regulate expression of the small temporal RNAs that control C. elegans developmental timing. Cell 106, 23-34 (2001).

36. Volpe, T. A. et al. Regulation of heterochromatic silencing and histone $\mathrm{H} 3$ lysine- 9 methylation by RNAi. Science 297, 1833-1837 (2002).

37. Mochizuki, K., Fine, N. A., Fujisawa, T. \& Gorovsky, M. A. Analysis of a piwi-related gene implicates small RNAs in genome rearrangement in Tetrahymena. Cell 110, 689-699 (2002).

38. Waterhouse, P. M., Graham, M. W. \& Wang, M. B. Virus resistance and gene silencing in plants can be induced by simultaneous expression of sense and antisense RNA. Proc. Natl Acad. Sci. USA 95, 13959-13964 (1998).

39. Dalmay, T., Hamilton, A., Rudd, S., Angell, S. \& Baulcombe, D. C. An RNA-dependent RNA polymerase gene in Arabidopsis is required for post-transcriptional gene silencing mediated by a transgene but not by a virus. Cell 101, 543-553 (2000)

40. Mourrain, P. et al. Arabidopsis SGS2 and SGS3 genes are required for post-transcriptional gene silencing and natural virus resistance. Cell 101, 533-542 (2000).

41. Cogoni, C. \& Macino, G. Gene silencing in Neurospora crassa requires a protein homologous to RNA dependent RNA polymerase. Nature 399, 166-169 (1999).

42. Sijen, T. et al. On the role of RNA amplification in dsRNA-triggered gene silencing. Cell 107, 465-476 (2001).

43. Smardon, A. et al. EGO-1 is related to RNA-directed RNA polymerase and functions in germ-line development and RNA interference in C. elegans. Curr. Biol. 10, 169-178 (2000).

44. Schiebel, W., Haas, B., Marinkovic, S., Klanner, A. \& Sanger, H. L. RNA-directed RNA polymerase from tomato leaves. II. Catalytic in vitro properties. J. Biol. Chem. 268, 11858-11867 (1993).

45. Makeyev, E. V. \& Bamford, D. H. Cellular RNA-dependent RNA polymerase involved in posttranscriptional gene silencing has two distinct activity modes. Mol. Cell 10, 1417-1427 (2002).

46. Dougherty, W. G. \& Parks, T. D. Transgenes and gene suppression: telling us something new? Curr. Opin. Cell Biol. 7, 399-405 (1995).

47. Baulcombe, D. C. RNA as a target and an initiator of post-transcriptional gene silencing in transgenic plants. Plant Mol. Biol. 32, 79-88 (1996).

48. Newport, J. Nuclear reconstitution in vitro: stages of assembly around protein-free DNA. Cell 48, 205-217 (1987).

49. Tabara, H., Yigit, E., Siomi, H. \& Mello, C. C. The dsRNA binding protein RDE-4 interacts with RDE-1, DCR-1, and a DExH-box helicase to direct RNAi in C. elegans. Cell 109, 861-871 (2002).

50. Dernburg, A. F., Zalevsky, J., Colaiacovo, M. P. \& Villeneuve, A. M. Transgene-mediated co-suppression in the C. elegans germ line. Genes Dev. 14, 1578-1583 (2000).

51. Ketting, R. F. \& Plasterk, R. H. A genetic link between co-suppression and RNA interference in C. elegans. Nature 404, 296-298 (2000).

52. Wassenegger, M., Heimes, S., Riedel, L. \& Sanger, H. L. RNA-directed de novo methylation of genomic sequences in plants. Cell 76, 567-576 (1994).

53. Mette, M. F., van der Winden, J., Matzke, M. A. \& Matzke, A. J. Production of aberrant promoter transcripts contributes to methylation and silencing of unlinked homologous promoters in trans. EMBO J. 18, 241-248 (1999). 
54. Reinhart, B. J. \& Bartel, D. P. Small RNAs correspond to centromere heterochromatic repeats. Science 297, 1831 (2002).

55. Pal-Bhadra, M. et al. Heterochromatic silencing and HP1 localization in Drosophila are dependent on the RNAi machinery. Science 303, 669-672 (2004).

56. Ketting, R. F., Haverkamp, T. H., van Luenen, H. G. \& Plasterk, R. H. mut-7 of C. elegans, required for transposon silencing and RNA interference, is a homolog of Werner syndrome helicase and RNaseD. Cell 99, 133-141 (1999).

57. Sijen, T. \& Plasterk, R. Transposon silencing in the Caenorhabditis elegans germ line by natural RNAi. Nature 426, 310-314 (2003).

58. Llave, C., Xie, Z., Kasschau, K. D. \& Carrington, J. C. Cleavage of Scarecrow-like mRNA targets directed by a class of Arabidopsis miRNA. Science 297, 2053-2056 (2002).

59. Tang, G., Reinhart, B. J., Bartel, D. P. \& Zamore, P. D. A biochemical framework for RNA silencing in plants. Genes Dev. 17, 49-63 (2003).

60. Yekta, S., Shih, I. H. \& Bartel, D. P. MicroRNA-directed cleavage of HOXB8 mRNA. Science 304, 594-596 (2004).

61. Palauqui, J. C., Elmayan, T., Pollien, J. M. \& Vaucheret, H. Systemic acquired silencing: transgenespecific post-transcriptional silencing is transmitted by grafting from silenced stocks to non- silenced scions. EMBO J. 16, 4738-4745 (1997).

62. Voinnet, O. \& Baulcombe, D. C. Systemic signalling in gene silencing. Nature 389, 553 (1997).

63. Storz, G. An expanding universe of noncoding RNAs. Science 296, 1260-1263 (2002).

64. Morey, C. \& Avner, P. Employment opportunities for non-coding RNAs. FEBS Lett. 567, 27-34 (2004).

65. Kiss, T. Small nucleolar RNAs: an abundant group of noncoding RNAs with diverse cellular functions. Cell 109, 145-148 (2002).

66. Wutz, A. \& Jaenisch, R. A shift from reversible to irreversible X inactivation is triggered during ES cell differentiation. Mol. Cell 5, 695-705 (2000).

67. Sleutels, F., Zwart, R. \& Barlow, D. P. The non-coding Air RNA is required for silencing autosomal imprinted genes. Nature 415, 810-813 (2002).

Acknowledgements C.C.M. is an HHMI Assistant Investigator and is funded by the NIH D.C. is supported by an NRSA postdoctoral fellowship.

Competing interests statement The authors declare that they have no competing financial interests. 